9. Kozar, K. et al. Cell 118, 477-491 (2004).

10. Tetsu, O. \& McCormick, F. Cancer Cell 3, 233-245 (2003).

11. Du, J. et al. Cancer Cel/ 6, 565-576 (2004)

12. Pagano, M. \& Jackson, P.K. Cell 118, 535-538 (2004)
13. Bartek, J., Lukas, C. \& Lukas, J. Nature Rev. Mol. Cell Biol. 5, 792-804 (2004).

14. Xiong, Y., Zhang, H. \& Beach, D. Cell 71, 505-514 (1992).

15. Malumbres, M. et al. Cell 118, 493-504 (2004).

16. Cheng, A., Xiong, W., Ferrell, J. E. Jr \& Solomon, M. J.
Cell Cycle 4, 155-165 (2005).

17. Dinarina, A. et al. Biochem. J. 386, 349-355 (2005).

18. Van Regenmortel, M. H. V. EMBO Rep. 5, 1016-1020 (2004).

\section{MicroRNAs diversify in Drosophila development}

MicroRNAs (miRNAs) have a unique way of preventing gene expression: by annealing with a target messenger RNA, they can inhibit its translation or even trigger its degradation. We are only just beginning to learn about their biological functions in animals, and Leaman et al. (Cell 121, 1097; 2005) have now conducted a screen in Drosophila embryos to reveal the broad developmental repertoire miRNAs are responsible for.

The approach taken by Leaman et al. was to inject early embryos with 2'-O-methyl antisense oligoribonucleotides and thereby deplete specific miRNAs. These oligoribonucleotides have been successfully applied before to inhibit small RNAs in vitro; they are thought to hybridize with the endogenous miRNA and so inhibit their action. In the new study, Leaman et al. took care to test whether these oligoribonucleotides can be used in the whole embryo with the same success and, through their controls, provided good support that they can and that specific inhibition can be achieved.

Using this approach, they systematically depleted embryos of 46 miRNAs expressed early in development. Of these, over half showed severe developmental phenotypes. The fact that loss of single miRNAs so often disrupted development suggests that they have distinct, nonredundant functions. An interesting observation was that compared to unique miRNAs, those that occur in multiple copies or within families were expressed more broadly and also showed more general, but readily discernible, defects.

From the range of phenotypes seen, it is clear that control by miRNAs is a key aspect of gene regulation in diverse developmental contexts. While $m i R-9$ is critical for cellularization, $m i R-31$ is needed for normal embryo segmentation. Later in development, several members of the $m i R-310$ family each seem to be required during the morphogenetic process of dorsal closure; importantly, the phenotypes for different family members are distinct.

Apoptosis is an integral part of normal development, and miRNAs have previously been linked to this pathway in Drosophila, at least during post-embryonic stages. Leaman et al. found that all four distinct miRNAs from the miR-2 family control apoptosis during embryogenesis, and so decided to take a closer look at what their targets might

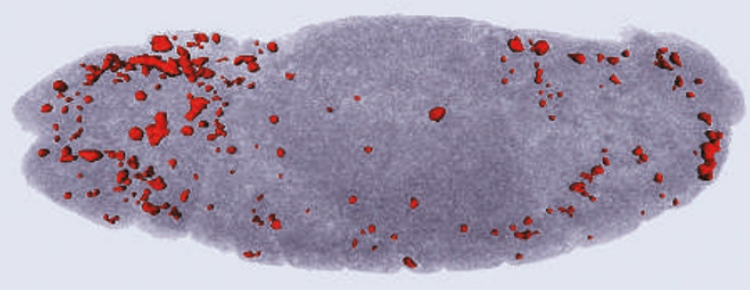

Wild type

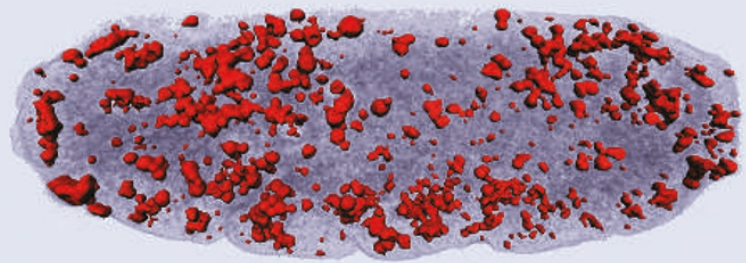

miR-6 antisense

miR-2 family microRNAs suppress apoptosis in Drosophila embryos. Three-dimensional reconstruction of an embryo injected with 2'-O-methyl antisense oligoribonucleotides to inhibit activity of the microRNA miR-6; staining with anti-caspase- 3 antibody (red) reveals massive cell death throughout the embryo. Image courtesy of Dan Leaman and Ulrike Gaul.

be. Previous studies using computational techniques predicted that the miR-2 family might bind to mRNAs encoding pro-apoptotic factors of the grim/reaper/hid family. Leaman et al. now confirm that the miR-2 miRNAs need these pro-apoptotic factors for their effects on apoptosis. They find that $m i R-2 \mathrm{~s}$ directly target these pro-apoptotic factors to mediate their translational repression, and that the $3^{\prime}$ end of each miRNA is key for determining target specificity.

The results from this screen provide solid grounds on which to build an understanding of how each of these miRNAs impinges on development. As demonstrated for the case of the $m i R-2$ family and apoptosis, this antisense technique also provides a convenient tool that can be used to test which developmental factors miRNAs target in each case.

ALISON SCHULDT 